\title{
Non-Hodgkin-Lymphom der Prostata
}

\author{
M. H. Djamilian, M. C. Truß, H. Krah, R. Schlick, M. A. Kuczyk, Ch. G. Stief, E. P. Allhoff \\ Urologische Klinik (Leiter: Prof. Dr. med. U. Jonas) Medizinische Hochschule Hannover
}

\section{Zusammenfassung}

Infiltrate maligner Lymphome sind in der Prostata sehr selten. Seit der ersten Beschreibung von Coupland 1877 (3) ist in der Literatur bisher von lediglich 30 Fällen berichtet worden. Wir berichten im folgenden über einen Patienten mit obstruktiven Miktionsbeschwerden und Non-Hodgkin-Lymphom, bei dem es während der transurethralen Resektion zu ausgeprägten und diffusen Blutungen kam, so da $\beta$ eine offene Revision nötig wurde. Präoperativ bestand weder klinisch noch sonographisch ein Anhalt für eine Infiltration in die Prostata. An eine Infiltration eines Non-Hodgkin-Lymphoms in die Prostata sollte bei entsprechender Anamnese auch bei negativem präoperativen Staging gedacht werden. Aufgrund des Blutungsrisikos sollten zunächst minimalinvasive Behandlungsmethoden angewandt werden.

\section{Non-Hodgkin-Lymphoma of the Prostate}

Infiltrates of malignant lymphoma in the prostate are rare. Since the first description by Coupland in 1877 (3), only 30 cases have been published. We report a case of a Non-Hodgkin-lymphoma with infiltration of the prostate presenting with symptoms of bladder outlet obstruction. During transurethral resection profound and diffuse bleeding occurred making immediate open revision necessary. Preoperatively, there was no clinical or ultrasonographic evidence for infiltration of the lymphoma into the prostate. In patients presenting with bladder outlet obstruction and with a history of lymphoma, possible infiltration into the prostate should be part of the differential diagnosis. Because of the increased risk of bleeding, minimal invasive treatment options should be considered first.

\section{Key words}

Prostate - Non Hodgkin lymphoma - Urinary obstruction - Complication - Hemorrhage

\section{Einleitung}

Das Prostatakarzinom metastasiert typischerweise in Lymphknoten und in das Skelettsystem, insbesondere in die Wirbelsäule. Seltener sind Metastasen in Leber und Lunge. Dagegen sind Metastasen und Infiltrate maligner Tumoren innerhalb der Prostata ausgesprochene Raritäten. Die Kasuistiken betreffen überwiegend junge Männer mit hochmalignen Non-Hodgkin-Lymphomen, deren Prognose schlecht ist $(1,2,5,7,9,12)$.

\section{Kasuistik}

Ein 70jähriger Patient wurde unserer Klinik wegen zunehmender obstruktiver Miktionsbeschwerden zugewiesen. Bei der Aufnahme klagte der Patient über eine stündliche Nykturie sowie über einen stark abgeschwächten Harnstrahl sowie Startschwierigkeiten und Nachträufeln. Die Anamnese ergab keine signifikanten Vorerkrankungen. Bei der rektalen Untersuchung fand sich eine etwa um das Doppelte vergrößerte Prostata von weicher Konsistenz ohne suspekte Areale. Eine transrek-

Akt. Urol. 25 (1994) 315-317

(C) Georg Thieme Verlag Stuttgart · New York tale Ultraschalluntersuchung zeigte eine gut abgrenzbare Prostata mit einzelnen Verkalkungszonen und einem Volumen von $40 \mathrm{ml}$. Suspekte Läsionen im Sinne von Hypodensitäten oder hyperechogenen Arealen waren nicht nachweisbar. Das prostataspezifische Antigen (PSA) wurde mit $2,1 \mathrm{ng} / \mathrm{ml}$ und die saure Prostataphosphatase (PAP) mit $0,8 \mathrm{ng} / \mathrm{ml}$ bestimmt. Es bestand somit kein Hinweis auf ein Prostatakarzinom oder eine Tumorinfiltration der Prostata. Der weitere körperliche Status war altersentsprechend normal, insbesondere bestand keine Hepatosplenomegalie.

Bei der Kontrolle der Routinelaborparameter fiel eine Leukozytose von 33000 Leukozyten/ $\mu \mathrm{l}$ mit 78 \% Lymphozyten auf. Daraufhin wurde die Diagnose eines malignen Non-Hodgkin-Lymphoms niedrigen Malignitätsgrades, entsprechend einer chronischen lymphatischen Leukämie, durch Knochenmarksbiopsie gesichert. Nach abgeschlossener präoperativer Diagnostik wurde eine transurethrale Prostataresektion unter der Verdachtsdiagnose einer benignen Prostatahyperplasie durchgeführt. Aufgrund von ausgeprägten Blutungen, verbliebenen Adenomresten und eines drohenden TUR-Syndromes wurde eine sofortige offene Revision durchgeführt. Der postoperative Verlauf war komplikationslos und der Pa- 


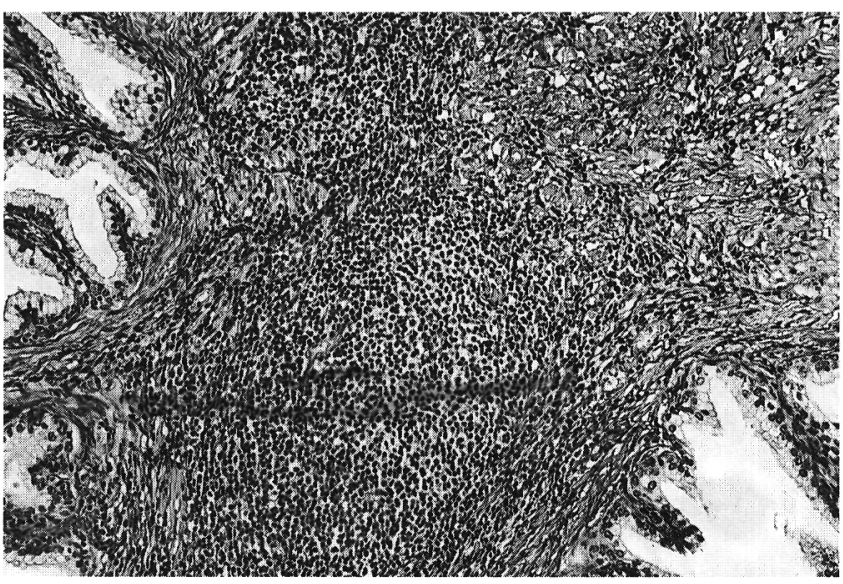

Abb. 1 Infiltration der Prostata durch ein lymphozytisches Non-Hodgkin-Lymphom (dunkelkernige Zellen in Bildmitte). Van Gieson-Färbung Vergrößerung $140 \times$

tient konnte mit deutlich verbesserter Uroflowmetrie und restharnfreier Blasenentleerung entlassen werden. Die weitere Therapie des Non-Hodgkin-Lymphoms erfolgte ambulant durch den Hausarzt des Patienten.

Die histologische Aufarbeitung des Präparates ergab eine noduläre und adenomatöse Hyperplasie der Prostata mit interstitiellen Infiltraten eines kleinzelligen, lymphozytischen Non-Hodgkin-Lymphoms (Abb. 1). Immunhistochemische Untersuchungen erbrachten stark positive Reaktionen der Lymphom-Infiltrate mit dem Leukozyten-Marker "Leucocyte Common Antigen" (CD45) sowie den B-Zell-Markern CD45RA (MB1) und CD45R (4KB5), während bei den T-Zell-Markern CD45RO (UCHL1) nur einzelne eingestreute reaktive T-Zellen und bei CD43 (MT1) keine positiven Zellen zu beobachten waren (Abb. 2).

\section{Diskussion}

Maligne Lymphome der Prostata sind Raritäten. Freeman et al. (4) fanden bei 1467 Patienten mit extranodalen Non-Hodgkin-Lymphomen nur $0,2 \%$ in der Prostata lokalisiert. Rainwater und Barrett (10) beschrieben erstmals ein primäres Lymphom in der Prostata, das durch transrektale Punktion eines sonographisch hypodensen Areals nachgewiesen wurde. Im vorliegenden Fall konnten im Gegensatz dazu keine hypodensen Areale gesehen werden, sonographisch sprach der Befund für das Vorliegen einer benignen Prostatahyperplasie. Trotz des seltenen Vorkommens müssen maligne Lymphome in die Differentialdiagnose der Prostatavergrößerung mit einbezogen werden. Sonographisch können diese als hypo- oder isodense Areale imponieren.

Bei 0,5 bis $1 \%$ der lymphoproliferativen Erkrankungen werden Thrombozytenfunktionsstörungen und verkürzte Thrombozytenüberlebenszeiten mit Thrombozytopenien, petechialen Blutungen und Hämorrhagien beobachtet $(6,7,11)$. Im vorliegenden Fall bestand keine Thrombozytopenie und die routinemäßig durchgeführten plasmatischen Gerinnungstests ergaben Normwerte. $\mathrm{Zu}$ sätzlich zu der Bestimmung der plasmatischen Routinege-

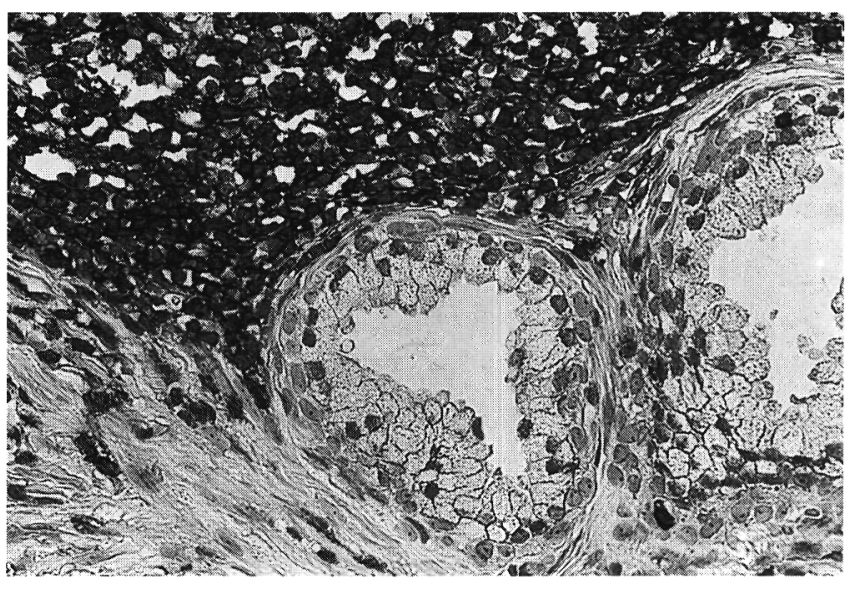

Abb. 2 Positive Reaktion der Lymphomzellen mit hier dunkler Markierung des Zytoplasmas nach Inkubation mit dem B-Zell-Marker CD45R. APAAP-Methode: Vergrößerung $360 \times$

rinnungsparameter sollte bei Patienten mit lymphoproliferativen Erkrankungen aufgrund des erhöhten Blutungsrisikos ein kompletter Gerinnungstest (Bestimmung der Gerinnungsfaktoren, Blutungszeit) erhoben werden. Weiterhin sollte die Indikation zu einer transurethralen Resektion oder einer offenen Adenomenukleation kritisch gestellt und nach Möglichkeit minimalinvasiveren Verfahren mit geringerem Blutungsrisiko (z. B. transurethrale Laserablation) der Vorzug gegeben werden.

\section{Literatur}

1 Banavali, S. D., K. M. Mohandas, R. Iyer, R. Gopal, T. K. Saikis, J. N. Kulkarni, C. S. Krishnamurthy, S. C. Soman, S. H. Advani: Non-Hodgkin's Lymphoma of prostate: a rare site of primary extranodal presentation (a report of two cases). Indian J. Cancer 28 (1991) 70-74

2 Bumpus, H. C.: Carcinoma of the prostate: a clinical study of one thousand cases. Surg. Gynecol. Obstet. 43 (1926) 150-155

3 Coupland, S.: Lymphoma (lympho-sarcoma) of the prostate: secondary nodules in pancreas and suprarenal capsule. Trans. Pathol. Soc. Lond. 28 (1877) 179

4 Freeman, C., J. W. Berg, S. J. Cutler: Occurrence and prognosis of extranodal lymphomas. Cancer 29 (1972) 252-260

${ }^{5}$ Heaney, J. A., R. A. Delellis, R. A. Rudders: Non-Hodgkin-Lymphoma arising in the lower urinary tract. Urology 25 (1985) $479-484$

6 Jones, $S$. E.: Autoimmune disorders and malignant lymphoma. Cancer 31 (1973) 1092-1098

${ }^{7}$ Kaden, B. R., W. F. Rosse, T. W. Hauch: Immune thrombocytopenia in lymphoproliferative diseases. Blood 53 (1979) $545-551$

8 Melicow, M. M., T. H. Pelton, G. W. Fish: Sarcoma of the prostate gland: review of literature; table of classification; report of four cases. J. Urol. 49 (1943) 675-707

9 Patel, D. R., G. A. Gomez, E. S. Henderson, M. Gamarra: Primary prostatic involvement in Non-Hodgkin-Lymphoma. Urology 32 (1988) 96-98

10 Rainwater, L. M., D. M. Barrett: Primary lymphoma of the prostate: transrectal ultrasonic appearance. Urology $36 / 6$ (1990) 522-525

11 Shulman, N. R., J. V. Jordan Jr.: Platelet Immunology. In: Colman, R. W., J. Hirsh, V. J. Marder, E. W. Salzman (eds.): Hemostasis and Thrombosis. Basic principles and clinical practice. Chapter 27, $2^{\text {nd }}$ edition. J. B. Lippincott Company, Philadelphia, 1987, p 509 
12 Suzuki, H., T. Nakada, Y. Iijima, H. Kaneko, Y. Suzuki, N. Ishii, Y. Onmura, A. Takamizawa, $M$. Saito: Malignant lymphoma of the prostate. Urol. Int. 47 (1991) 179-185

\section{Dr. M. H. Djamilian}

\section{Urologische Klinik}

Medizinische Hochschule Hannover

Konstanty-Gutschow-Straße 8

30623 Hannover

\section{Kommentar}

Die Autoren beschreiben einen Fall einer Lymphommanifestation innerhalb der Prostata im Rahmen einer chronisch lymphatischen Leukämie (CLL). Aufgrund einer nach Operation aufgetretenen Blutungskomplikation schließen die Autoren auf eine erhöhte Blutungsgefahr in diesem Patientenkollektiv und warnen vor unkritischer Indikationsstellung in solchen Fällen.

Die chronisch lymphatische Leukämie ist eine klonale lymphoproliferative Erkrankung mit sehr variablem Verlauf. Es werden sowohl relativ aggressive Verläufe mit 1-2 Jahren Überlebenszeit als auch extrem indolente, benigne Varianten mit Überlebenszeiten bis zu 20 Jahren beobachtet. Eine extralymphatische Krankheitsmanifestation ist nicht als unabhängiger Prognosefaktor etabliert.

Blutungsneigung kann bei CLL-Patienten aus mehreren Gründen entstehen. Die häufigste Ursache stellen Thrombozytopenien dar. Diese können durch Thrombozytenbildungsstörungen wegen der Knochenmarksinfiltration und/oder autoimmun durch vermehrten Abbau verursacht sein. Selten kommen bei dieser Erkrankung monoklonale Gammopathien vor. In Einzelfällen wurde diesen pathologisch vermehrten klonalen Immunglobulinmolekülen eine inhibitorische Aktivität gegenüber Gerinnungsfaktoren oder der Thrombozytenfunktion zugeschrieben. Dies kann zu klinisch bedeutsamen Gerinnungsstörungen führen.

$\mathrm{Zu}$ potentiellen Mechanismen der Gerinnungsstörung im vorliegenden Fall wird von den Autoren nicht Stellung bezogen. Da Gerinnungsstörungen bei normaler Thrombozytenzahl bei dieser Erkrankung jedoch selten sind, erscheint es nicht sinnvoll, im Rahmen der präoperativen Untersuchung bei allen CLL-Patienten alle Gerinnungsfaktoren zu bestimmen. Solch weiterführende Tests sind auch bei CLL nur bei pathologischen Gerinnungswerten und/oder Blutungszeit indiziert. Über die Indikation zu invasiven Eingriffen muß im Einzelfall aufgrund der Beschwerden der Patienten, der Prognose der individuellen Erkrankung und Therapiealternativen wie Chemotherapie oder lokale Bestrahlung entschieden werden. Dabei muß bedacht werden, daß in diesem Patientenkollektiv meist vorgerückten Alters die beschriebene Inzidenz von Lymphommanifestationen in der Prostata von $0,2 \%$ immer noch deutlich niedriger ist als die erwartete Inzidenz von Prostatahypertrophie.

PD Dr. med. Walter Aulitzky

Abteilung Hämatologie

III. Medizinische Klinik

Klinikum der Johannes Gutenberg-Universität

Langenbeckstr. 1

55131 Mainz 